\title{
Hierarchical multi-reservoir optimization modeling for real-world complexity with application to the Three Gorges System
}

\author{
Fang-Fang Li ${ }^{\mathrm{a},{ }^{*}}$, Christine A. Shoemaker ${ }^{\mathrm{b}}$, Jun Qiu ${ }^{\mathrm{c}}$, Jia-Hua Wei ${ }^{\mathrm{d}}$ \\ ${ }^{a}$ College of Water Resources \& Civil Engineering, China Agricultural University, Beijing \\ 100083,China,liff03@gmail.com \\ ${ }^{b}$ School of Civil \&Environmental Engineering, Cornell University, Ithaca, NY, USA \\ ${ }^{c}$ Institute for Aero-Engine, School of Aerospace Engineering, Tsinghua University, \\ China \\ ${ }^{d}$ State Key Laboratory of Hydroscience \& Engineering, Tsinghua University, Beijing, \\ China
}

\begin{abstract}
High dimensionality in real-world multi-reservoir systems greatly hinders the application and popularity of evolutionary algorithms, especially for systems with heterogeneous units. An efficient hierarchical optimization framework is presented for search space reduction, determining the best water distributions, not only between cascade reservoirs, but also among different types of hydropower units. The framework is applied to the Three Gorges Project (TGP) system and the results demonstrate that the difficulties of multi-reservoir optimization caused by high dimensionality can be effectively solved by the proposed hierarchical method. For the day studied, power output could be increased by $6.79 \mathrm{GWh}$ using an optimal decision with the same amount of water actually used; while the same amount of power could be generated with $2.59 \times 10^{7}$ $\mathrm{m}^{3}$ less water compared to the historical policy. The methodology proposed is general in that it can be used for other reservoir systems and other types of heterogeneous unit generators.
\end{abstract}

Keywords-Hierarchical optimization; heterogeneous hydropower units; multireservoirs; heuristic algorithms; the Three Gorges Project

\section{Introduction}

Although metaheuristics have been used extensively in the water resources field due to their ability to link with simulation models, their application to real-world problems 
raises a number of methodological challenges as stated by Maier et al. (2014). The high dimensionality of the decision variables in real-world problems contributes a lot to these application challenges, such as problem formulation, size of search space, computational efficiency and so on.

Reservoir optimization is one of the application areas of metaheuristics. Optimization of multiple hydropower facilities with heterogeneous hydropower units is important throughout the world. However, both the non-linearity existing widely in the multi-reservoir system and the high-dimensionality resulting from multiple reservoirs and the heterogeneous units lead to difficulties for the optimization.

There have been numerous techniques reported in the literature to optimize reservoir operation. Labadie (2004) presents an overview on the methods used for this problem, mainly including linear programming (LP), nonlinear programming (NLP), dynamic programming (DP) and heuristic optimization. The complex hydraulic and electrical interaction among reservoirs makes heuristic algorithms popular for multi-reservoir optimization. Various heuristic algorithms have been applied, such as Simulated Annealing (SA) (Tospornsampan et al., 2005; Wong and Wong, 1994), Evolutionary Programming (EP) (Yang et al., 1996), Genetic Algorithm (GA) (Arce et al., 2002; Gil et al., 2003; Malekmohammadi et al., 2009; Orero and Irving, 1998), Artificial Neural Network (ANN) (Naresh and Sharma, 2000), and methods based on response surface (Castelletti et al., 2010).

To reduce the size of the search space and simplify the problem, the majority of the research on multi-reservoir optimization treats the hydropower plant as a whole, without consideration of the heterogeneous characteristics of the hydropower units. Even for those studies on short-term hydro scheduling, since they are often coordinated with thermal power plants (Barros et al., 2003; Cai et al., 2001; Grygier and Stedinger, 1985; Yeh et al., 1992), the operation of hydro power station is often simplified to enhance the solution speed at the expense of accuracy. The problem of optimal scheduling heterogeneous units is either partially addressed or not addressed at all (Ponrajah et al., 1998). When considering variations among different types of hydropower units, mathematical programming models with hierarchical ideal are the most frequent approaches for hydro scheduling problem. Lagrangian Relaxation (LR) was once considered to be the best approach to deal with large-scale unit commitment, which 
supplies good starting points for heuristic searches to obtain feasible primal solutions. Nevertheless, researchers acknowledge that it cannot guarantee the optimal solution (Duo et al., 1999), and the dualization schemes are problem dependent (Finardi and Scuzziato, 2014). Mixed integer linear programming (MILP) based methods have also been popular for short-term hydro generation scheduling in recent years, which calls for piecewise linear approximation of the forebay level, tailrace level, penstock loss, and hydropower production functions (Borghetti et al., 2008). However, Tong et al. (2013) found that such linearization may result in infeasible solutions. Yi et al.(2003) also conclude that, compared with MILP, DP has better basin operating efficiency, faster execution time, and robust performance; and most importantly, DP algorithm execution time increases approximately linearly with additional units, whereas other methods experience exponential increases in computation time. Unfortunately, DP is unsuitable for Hydro Electric Commission (HEC) optimal hydro scheduling problems in multi-reservoir systems because of high computational requirements and the difficulty of incorporating coupling constraints between reservoirs (Piekutowski et al., 1994). Some other approaches utilizing decomposing or layering are also proposed for multi-reservoir systems (Saad et al., 1996; Wakamori et al., 1982), while unit scheduling is still not taken into account for the purpose of dimensionality reduction.

From a physical perspective, the optimization of multi-reservoir systems with heterogeneous units can be divided into two levels: (1) to determine the optimal water dispatching scenario among different reservoirs; and (2) for each reservoir, to decide the best hydro scheduling plan of different hydropower units, including unit commitment and optimal load dispatch with the given hydraulic condition.

In this paper, a hierarchical model for short-term operation of multi-reservoir power systems with heterogeneous hydropower units is presented. Since for short-term hydro scheduling, the load curve is given by the power grid, the objective function is to minimize the amount of water used to satisfy the load demand, as well as other practical constraints. We also consider an alternative objective, which is to maximize the power output with the actual amount of water used historically. There are two loops in the hierarchical model: the outer loop uses heuristic algorithms to distribute water among reservoirs, and the inner loop adopts linear programming to dispatch water to different types of units in the most efficient way. The choice of heuristic algorithms for the outer 
loop is difficult, as there are many choices and heuristic optimization tends to require a large number of evaluations. Seven typical heuristic algorithms with different designed ideas are compared for the outer loop, including: Genetic Algorithms (GA), Particle Swarm Optimization (PSO), Simulated Annealing (SA), Dynamically Dimensioned Search (DDS), DYnamic COordinate search using Response Surface models (DYCORS), and Stochastic Radial Basis Function method (SRBF). Given that GA approaches are well established, we considered both a Simple GA (SGA) and an Improved GA (IGA). Each evaluation of the heuristic optimizer in the outer loop requires solving the inner loop problem by linear programming, which is highly efficient, even with a large number of variables.

Such a hierarchical approach reduces computational effort by greatly decreasing the number of variables that must be analyzed by a heuristic algorithm. Also, because the outer loop incorporates a small number of variables that are linked between time periods, the inner loop can solve individual optimization problems for each time step, which is less computationally expensive than solving one large multi-time period optimization problem. Although multi-level optimization has perhaps been suggested in a few other disciplines, the challenge is to conceptualize how to formulate a specific problem into levels so that one can preserve optimality while creating multiple levels of optimization that will be much more efficient than single level optimization.

The Three Gorges Project (TGP) is the one of the largest hydropower plants in the world, with an installed power generating capacity of $18,200 \mathrm{MW}$. Together with Gezhouba reservoir (GZB) $38 \mathrm{~km}$ downstream, TGP plays an indispensable role in China's strategy for energy. Completed in 17 years in three phrases, the hydropower units installed in TGP are complex. In addition, the planning for the TGP began nearly 40 years after construction of the GZB, and the units of the GZB had to be upgraded to meet the water head change after the construction of the TGP, thus the units designed in the GZB are also complicated.

Taking TGP-GZB cascade hydro power station as an example, this study not only applies the proposed hierarchical model, but also tests different heuristic algorithms for the outer loop. Two ways to generate initial populations are considered. One is taking the actual operation as guidance, and the other is generating all solutions randomly. The characteristics of each kind of heuristic algorithm are analyzed to provide reference for 
algorithm selection. The efficiencies of different types of hydro units on a given day are also compared, as well as the performance of different algorithms in different seasons. The study demonstrates that the difficulties of multi-reservoir optimization caused by non-linearity and high-dimensionality can be effectively solved by the proposed hierarchical method, which can be used for other reservoir systems and other types of heterogeneous unit generators than those studied here.

\section{Methodology}

\subsection{Design thought of the hierarchical model}

Both the high-dimensionality and the nonlinearity of multi-reservoir systems contribute to the difficulties in calculation. Not only the hydraulic interaction between multiple reservoirs is nonlinear, but also the power output of hydropower unit has a constantly changing nonlinear relationship with different turbine discharge and water head, which itself is a nonlinear function of reservoir storage. These nonlinear relationships existing widely in multiple hydropower systems result in multiple local minima for the optimization problem, i.e. the optimization surface is multimodal. Hence, heuristic algorithms are required, since they do not have particular requests for the mathematical structure of the problem.

Fig. 1 shows an example of the power output characteristic curves of two different types of hydropower units in the Three Gorges Project (TGP) power station in China under the water head of $100 \mathrm{~m}$. In Fig. 1 with the same turbine discharge of $700 \mathrm{~m}^{3} / \mathrm{s}$, the Ha type of units can output $2.4 \%$ more power, which refers to $15 \mathrm{MW}$, but when there is a high flow at $900 \mathrm{~m}^{3} / \mathrm{s}$, the ALS type is more efficient, with $3.7 \%$ more power production. 


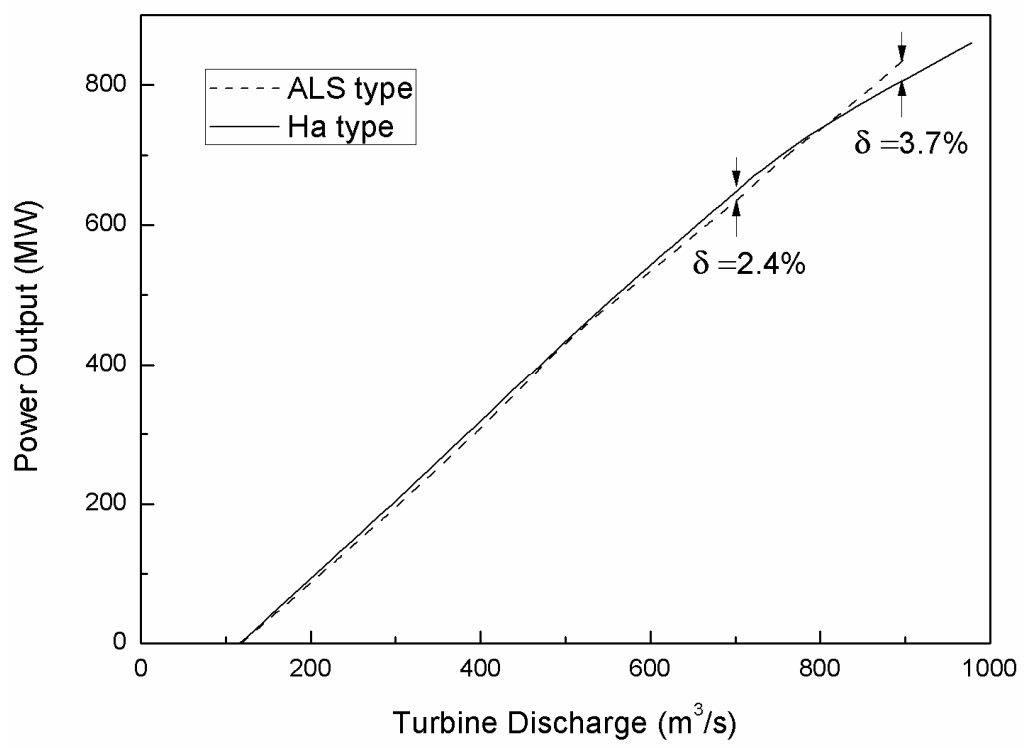

Fig.1. Power output characteristic curves of two types of hydropower units in the TGP power station under the water head of $100 \mathrm{~m}$

It can be seen from Fig. 1 that it is essential to consider variations among different types of hydropower units in order to distribute load demand in a more efficient way (Diniz et al., 2007). Nevertheless, when taking the differences of the units into account, another source of difficulties, the high dimensionality, rises (Sulis, 2009). For any hydropower scheduling problem, the dimension of decision variables is $m \times T$, with $m$ representing the total number of unit types, and $T$ the total number of time steps. For those reservoirs where the same type of units are installed, $m$ could be as small as 1, while for those with different types of units, the dimension of the decision variable could be very high. For a system with 10 types of generator units, even there are only 5 time intervals, the dimension of decision variables is as high as 50. Considering unit commitment, even larger search space is generated. Taking the TGP cascade system as an example, there are 10 different types of units with respective allowable numbers of integer values ranging from 0 to the maximum given in Section 3.1. Such a complex whole has caught $6.6 \times 10^{30}$ possible values of the integer vectors describing the selection of the numbers of units used in each of 5 time steps.

Unfortunately, heuristic optimization algorithms (e.g. genetic algorithms) can take an unfeasibly long time to find answers to continuous variable problems with many 
decision variables (Chang et al., 2010; Li et al., 2012). The main reason is that even if one element on the dimensions mutates into an inappropriate value, the whole solution becomes infeasible. During the iteration of the optimization, very few solutions generated randomly in search space are feasible.

As analyzed above, the non-linearity and the high dimensionality of the multi-reservoir systems lead to the requirement of (1) the application of the heuristic algorithms; and (2) reducing dimensionality.

With the purpose of dimensionality reduction, a hierarchical model is proposed, which decouples the optimization of multi-reservoir system into two loops: (1) outer loop: decide the best distributions of the water among different reservoirs, represented by water head $H_{r}^{t}$, and reservoir outflow $Q_{r}^{t}$; and (2) inner loop: for each reservoir, given the water dispatched in the outer loop, optimize the unit commitment and the load dispatch scheduling to produce power generation in the most efficient way. The inner loop decision variables of : (1) number of each type of units which should put into operation at $t$ (decision vector is $\left.\vec{n}^{t}=\left(n_{1}^{t}, n_{2}^{t}, \ldots, n_{m}^{t}\right)\right)$; and (2) turbine water discharge of units $i$ at $t$ (decision vector is $\vec{q}^{t}=\left(q_{1}^{t}, q_{2}^{t}, \ldots, q_{m}^{t}\right)$ ) are chosen to maximize power output from reservoir $r$ given $H_{r}^{t}, Q_{r}^{t}$ from the outer loop.

The outer loop requires heuristic optimization, and the inner loop with many decision variables can be solved by highly efficient linear programming. The candidate solutions of the outer loop, which are the water dispatch scenarios between reservoirs, provide the boundary condition for the inner loop. The solution of the inner loop, which is optimal hydro scheduling for each water dispatch scenarios, serves as the constraints for the outer heuristic optimization problem. There are also some other constraints on water level and release. Seasonal hydrology data such as inflow information is taken as input, and hourly allocation to hydropower units can be updated daily or more frequently.

\subsection{Power output characteristics}

A number of models (El-Hawary and Christensen, 1979) have been used to represent the power output of hydro units. The basic function to calculate the hydropower output $P$ is :

$$
P=K Q H
$$

where $K$ is the synthetic output coefficient; $Q$ is the reservoir outflow; and $H$ is the water 
head of the reservoir.

According to Eq. (1), once the value of water head $H$ is fixed, the power output $P$ of hydropower unit $i$ can be approximated by Eq. (2):

$$
P_{i}^{t}=C_{1 i}\left(H_{r}^{t}\right)^{\prime} q_{i}^{t}+C_{2 i}\left(H_{r}^{t}\right)
$$

where $q$ is the turbine water discharge of unit $i ; C_{l i}$ and $C_{2 i}$ are fit from the power output characteristic database as shown in Fig.1, $H_{r}^{t}$ is the water head of reservoir $r$ where unit $i$ are installed, calculated by Eq. (3):

$$
H_{r}^{t}=E L_{r}^{t}\left(V_{r}^{t}\right)-T L_{r}^{t}\left(Q_{r}^{t}\right)-H L_{r}^{t}\left(Q_{r}^{t}\right)
$$

where $E L$ is the water level; $V$ the storage of reservoir $r$ at $t ; T L$ the Tail water elevation ; $H L$ is the water head loss, which is from the database describing the relationship between water head loss and inflow; $r$ is the index of reservoirs, set at 1 and 2, with 1 standing for the Three Gorges Project (TGP) and 2 for the Gezhouba (GZB); and $t$ the index of time steps. For instance, $Q_{r}^{t}$ is the outflow of reservoir $r$ at time $t$.

The only precondition of such linearization is fixed water head. So we divide the water head of reservoirs into small ranges, and within each range, the water head is regarded to be constant.

\subsection{Hierarchical model for multi-reservoir system}

As described above, the optimization objective of the outer loop can be alternative. In this study, two popular objectives for short-term reservoir operation are studied, defined as: (1) Case 1: with objective of minimizing the amount of water used and (2) Case 2: with objective of maximizing the total power generation. However, in both cases, when considering the problem of how to dispatch water to the different hydropower units, the objective becomes to optimize the hydropower units commitment as well as the load dispatch among different types of units in the most efficient way.

There are essentially two hierarchical levels of the optimization, as illustrated in Fig.2. 


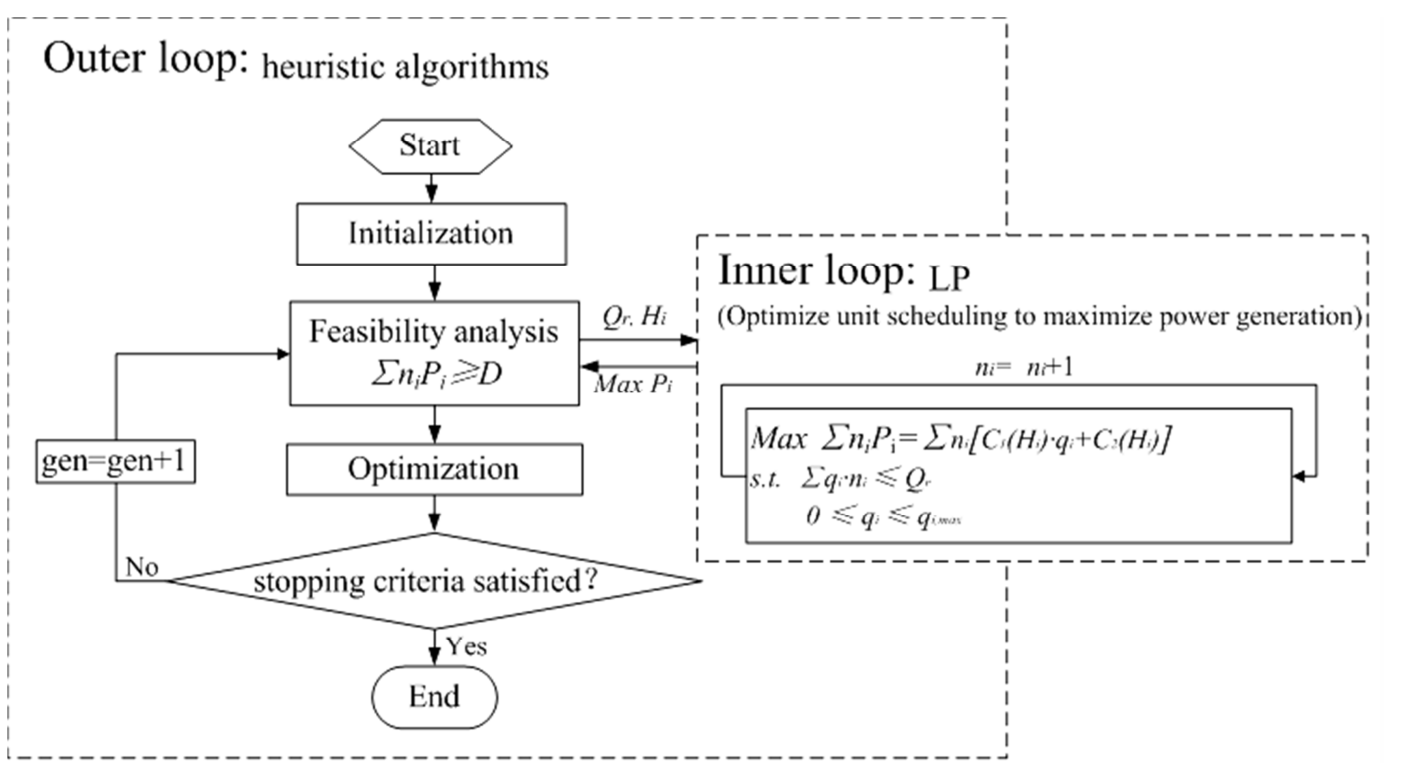

Fig.2. Flow chart of the bi-level optimization model

Fig. 2 shows the framework of the hierarchical optimization model presented in the paper. The outer loop of the optimization searches the optimal water allocation scenarios among the multiple reservoirs. First, the candidate scenarios are generated for the heuristic optimization methods. Then the feasibility of the scenarios are analyzed, in which, the power output has to be calculated. The inner loop is used to determine the optimal hydro scheduling, which gives out the optimal unit commitment and load dispatch by linear programming, as well as the corresponding maximum power output. After the feasibility analysis, the objective function values are calculated. Finally, heuristic search algorithms are used to identify the best solution. This is the optimal water allocation policy between different reservoirs and the corresponding optimal hydro unit scheduling. The iterations continue until the stopping criterion is satisfied, which is set to be 10,000 evaluations in this study.

\subsubsection{Inner loop optimization.}

The inner loop decides how to allocate water among $i=1,2, \ldots, m$ types of hydropower units to maximize the power output in each reservoir $r$, given that there is $Q_{r}^{t}$ volume of water, $\vec{n}^{t}$ (a vector of the number of each type of units used in period $t$ ), and the water head is $H_{r}^{t}$. Different types of units $i$ are receiving different turbine 
discharge described by the vector $\vec{q}^{t}$, which is a decision vector in the inner loop optimization. This can be described as a linear programming problem:

$$
\begin{aligned}
& \begin{aligned}
z_{r}^{t}\left(Q_{r}^{t}, \vec{n}^{t}, H_{r}^{t}, r\right) & =\operatorname{Max}_{\vec{q}^{t}} \sum_{i=1}^{m} n_{i}^{t} P_{i}^{t} \\
& =\operatorname{Max}_{\vec{q}^{t}} \sum_{i=1}^{m} n_{i}^{t}\left[C_{1 i}\left(H_{r}^{t}\right) \times q_{i}^{t}+C_{2 i}\left(H_{r}^{t}\right)\right]
\end{aligned} \\
& \text { s.t. } \sum^{m}\left(n_{i}^{t} \times q_{i}^{t}\right) \leq Q_{r}^{t}, r=1, \ldots, M \quad 0 \leq q_{i}^{t} \leq q_{i}^{\max }
\end{aligned}
$$

$z_{r}^{t}\left(Q_{r}^{t}, \vec{n}^{t}, H_{r}^{t}, r\right)^{i=1}=$ the maximum value of the objective function in Eq. (4) that can be obtained by Linear Programming by varying decision vector $\vec{q}^{t}$. The fixed values are $Q_{r}^{t}, \vec{n}^{t}$ and $H_{r}^{t}$.

The inner loop uses linear programming (specified by Eq. (4) to Eq. (5)) to find the best value of $\vec{q}^{t}$ when $Q_{r}^{t}, \vec{n}^{t}, H_{r}^{t}$ are fixed. This problem is solved repeatedly for different possible value of $\vec{n}^{t}$ to find the best value of $z_{t}\left(q_{i}^{t} \mid \vec{n}^{t}, H_{i}^{t}\right)$ in Eq. (4). So

$$
P_{r}^{* t}\left(Q_{r}^{t}, H_{r}^{t}, r\right)=\operatorname{Max}_{\vec{n}^{t}} z_{t}\left(Q_{r}^{t}, \vec{n}^{t}, H_{r}^{t}, r\right)
$$

$P_{t}^{*}\left(Q_{r}^{t}, H_{r}^{t}, r\right)$ is then the optimal value for power production computed by the inner loop for reservoir $r$ when $Q_{r}^{t}, \vec{n}^{t}, H_{r}^{t}$ are fixed. Then the outer loop (next section) searches for the best value of $Q_{r}^{t}$ and $H_{r}^{t}$ to optimize the objective function in Eq. (7) and Eq. (8) with heuristic algorithms. The results of the inner loop optimization serve as constraints for the outer loop.

A major component of the computationally efficient approach we propose for this problem is the choice of inner loop decision variables to be optimized in the inner loop. These were selected because they define a linear optimization problem that can be efficiently solved with linear programming when the outer loop decision variables are fixed. Some of the decision variables $Q_{r}^{t}, \vec{n}^{t}$ and $H_{r}^{t}$ have nonlinear relationships, and multiple local optimum are generated, thus the optimization methods needs to have (1) little requirement on the mathematical structure of the model, and (2) the ability to jump out of the local optimum. Such global optimization algorithms usually take a very large number of evaluations. The number of evaluations with heuristic optimization increases rapidly with the dimension of the decision vector, so the computation will be much more efficient with the bi-level approach proposed here that reduces the number of 
heuristic optimization variables by moving all the $\vec{q}^{t}$ out of the heuristic optimization problem.

\subsubsection{Outer loop objective function.}

The aim of the outer loop is to pick the optimal value of $Q_{r}^{t}$ for all $r$ and $t$, which is the total amount of water to be released from reservoir $r$ in period $t$, and as a result $H_{r}^{t}$ is obtained.

There are two alternative objectives usually considered in the operation of reservoir. One is to minimize the amount of water used in the time period while meeting demand. The other is to maximize the power generation produced by the released water. These two objectives contradict each other, which can be considered simultaneously by using multi-objective optimization. Such multi-objective optimization could support decision making with the predicted inflow and benefit the real-time operation. Since the motivation of this study is to estimate the actual operation with the historical inflow instead of decision making support, the maximal power generation with the same water used in actual operation is more concerned, as well as the minimal amount of water with the demand satisfied. In other words, we intend to spend calculation cost in improving the two endpoints of the Pareto Front of this multi-objective optimization problem, instead of getting the information of the whole Pareto Front. Also, different algorithms need to be evaluated for this hierarchical model. So these two objectives are considered alternatively in this paper.

Case 1 is to minimize the amount of water used to satisfy the load demand, as well as other physical, legal and environmental constraints. It should be noted that since the release from the reservoir upstream can be reused by the hydro power station downstream, the amount of water to be minimized refers to the water used in the first reservoir upstream of the multi-reservoir power system, which equals the release from the reservoir $Q_{1}^{t}$ times its duration time, as shown in Eq. (7). Case 2 is to maximize the total power generation of multi-reservoir power system with the actual amount of water available, shown in Eq. (8), which is also required to meet the load demand and other constraints. The objective functions are:

$$
\text { Case } 1 \quad G_{1}=\operatorname{Min} \sum_{t} Q_{1}^{t} \times \Delta t_{t}
$$




$$
\text { Case } 2 \quad G_{2}=\underset{Q_{r}^{t}}{\operatorname{Max}} \sum_{t} \sum_{r} P_{r}^{* t} \times \Delta t_{t}
$$

The objective functions of both cases are required to satisfy some constraints related to $Q_{1}^{t}$, which are contained in the outer loop decision vector $Q$. $Q$ affects power output $P^{*}$, which is optimized by linear programming as described in Eq. (4) to Eq. (5). The constrains are used to check if the total output can satisfy the load demand as shown later in Eq. (14) for both Case 1 and Case 2, and also contributes to the calculation of objective function value of Case 2 in Eq.(8). Now $G_{j}$ connects Eq. (4) to Eq. (7) and Eq. (8) with the value of $z$ being a solution in the inner loop and $G$ being a solution from the outer loop.

\subsubsection{Heuristic optimization algorithms for the outer loop}

Various heuristic algorithms have been reported, as well as their modified patterns. These algorithms can be simply classified into two groups: population-based algorithms and single point-based methods (also known as trajectory or local search methods) (Maier et al., 2014). According to the No Free Lunch (NFL) theorem by Wolpert and Macready (Wolpert and Macready, 1997), optimization algorithms cannot be universally good. Hence, only the original algorithms with different design concepts are selected to check out which train of thoughts is suitable for our 10 dimensional problem of reservoir optimization.

The seven kinds of different heuristic algorithms selected in this study includes: one of the most popular algorithm in the past (GA), another classical population-based evolutionary algorithm (PSO), the typical algorithm based on individual iteration (SA), a relatively new algorithm using individual iteration (DDS), and some frontier algorithms based on response surface developed recently (DYCORS and SRBF). Specifically, for the GA, both the Simple GA (SGA) and Improved GA (IGA) are considered.

\section{Simple genetic algorithm (SGA)}

Genetic Algorithm (GA) is a search algorithm based on the mechanism of natural selection and natural genetics, which originated in mid 1970s. The basic concept of GA is to simulate natural evolutionary processes, mainly including three stages: initialization, fitness computation and new population generation. Every new population is generated by three genetic operators: selection, crossover and mutation. With the roulette selection, 
those individuals with better objective functions values are more likely to be selected to participate in the next crossover and mutation. This process iterates for a certain number of generations until the termination criterion is satisfied.

\section{Improved genetic algorithm (IGA)}

There have been many improvements for GA to enhance its ability in heuristic search. Not only SGA but also IGA (Kirkpatrick, 1984) is tested in this research. Improved Genetic Algorithm (IGA) is similar to simple genetic algorithm except IGA replaces roulette with tournament selection and uses SBX-Crossover (Deb and Agrawal, 1995) and polynomial mutation (Deb and Goyal, 1996). Also IGA uses the constraint handling method suggested by Deb (Deb, 2000).

\section{Particle Swarm Optimization (PSO)}

The main idea of PSO (Kennedy and Eberhart, 1995) is to iteratively improve a candidate solution with regard to a given measure of quality. A population of candidate solutions dubbed particles keeps moving around in the search-space according to simple mathematical formulae over the particle's position and velocity. Each particle's movement is influenced by its local best known position, while also guided toward the best known positions in the search-space. The process is repeated and by doing so it is hoped, but not guaranteed, that a satisfactory solution will eventually be discovered.

\section{Simulated annealing $(S A)$}

In Simulated Annealing (SA) (Singh, 2011) a point is picked at random from a neighborhood around the current solution. If the new random point is 'accepted', it becomes the next current solution. The random point is accepted if its cost value is better than the cost value of the current solution or (if the cost value of the new random point is worse than the current solution) the random point can be accepted with a probability of $p$, where $p$ decreases as the difference ( $\Delta$ cost) between the cost values of the current and new random point increases. Hence, SA can move from a good solution to a worse solution with a probability. This is SA's strategy for not becoming caught in a local minimum. This probability $p$ is gradually decreasing as a function of $\Delta$ cost as the number of iterations increase due to decreases in the 'temperature' parameter $T$. Hence 
the probability of selecting a worse or 'uphill' solution (for a minimization problem) declines. The allowance for 'uphill' moves potentially saves the method from becoming stuck at local optimum.

\section{Dynamically dimensioned search (DDS)}

The Dynamically Dimensioned Search algorithm (DDS) (Tolson and Shoemaker, 2007) is a recently developed stochastic based heuristic search algorithm with a population size of one (like simulated annealing). The algorithm searches globally at the start of the search and becomes a more local search as the number of iterations approaches the maximum allowable number of function evaluations. The adjustment from global to local search is achieved by dynamically and probabilistically reducing the number of dimensions in the neighborhood. Candidate solutions are created by perturbing the current best solution values only in the limited number of randomly selected dimensions. These perturbation magnitudes are randomly sampled from a normal distribution with a mean of zero. The only algorithm parameter to set in the DDS algorithm is the scalar neighborhood size perturbation parameter $(r)$ that defines the standard deviation of the random perturbation size standard as a fraction of the decision variable range.

\section{DYnamic COordinate search using Response Surface models (DYCORS)}

The DYnamic COordinate search using Response Surface model (DYCORS) incorporates the idea from DDS with surrogate model (Regis and Shoemaker, 2013). The main structure of DYCORS is the same as many other response surface-based optimization methods. At first, the objective function at candidate points is evaluated to fit a response surface model. In each iteration, this surrogate model of the objective function is built and improved using a dynamic coordinate search strategy for generating trial solutions that is similar to the one used in DDS. Only a subset of the coordinates of the current best solution is perturbed, and also the probability of perturbing a coordinate decreases with the iterations. The radial basis function (RBF) interpolation model given in Regis and Shoemaker's study (Regis and Shoemaker, 2013) which was previously described in Powell (Powell, 1992), is used in the DYCORS algorithms in this study.

\section{Stochastic Radial Basis Function method (SRBF)}


The Stochastic Response Surface Method is a recent stochastic method for the global optimization using a response surface model. When used with an Radial Basis Function (RBF) surrogate model, it is called Stochastic RBF(Regis and Shoemaker, 2007). The Stochastic RBF (SRBF) iteratively utilizes a response surface model to approximate the objective function and identifies a promising point for function evaluation from a set of randomly generated points. To search on surrogate surface and find next evaluation points, many candidate points are generated by some random method. Only a subset of them are selected for real objective function evaluations, as those points are with the best weighted score from two criteria: (1) estimated function value obtained from the surrogate model, and (2) maxi mum distance from previously evaluated points. The RBF model used in this study is from Powell (Powell, 1992; Powell, 1999) and was used as the basis of the RBF method by Gutmann (Gutmann, 2001).

\subsection{Constraints}

Mathematically, the reservoir operation needs to satisfy various equality and inequality constraints, including:

\subsubsection{Water balance equality constraints}

Water balance Eq.s imply not only the hydraulic connection between the reservoir upstream and downstream, but also the hydraulic relations between neighboring time steps, so the reservoir volume $V_{r}^{t+1}$ is given below:

$$
V_{r}^{t+1}=V_{r}^{t}+I_{r}^{t} \times \Delta t_{t}-Q_{r}^{t} \times \Delta t_{t}
$$

where $I$ is the inflow to reservoirs.

\subsubsection{Water level-storage curves of the reservoirs}

A non-linear function $f(x)$ is adopted to express relationship between water level $E L_{r}^{t}$ and storage $V_{r}^{t}$.

$$
E L_{r}^{t}=f\left(V_{r}^{t}\right)
$$

\subsubsection{Tailwater elevation curves at reservoir}

Since the water level of the reservoir downstream might have upward-effect on the 
tail water elevation of the reservoir upstream, tailwater elevation curves represent the relationship among the tailwater elevation, the reservoir release, and the water level of the next reservoir downstream, which are described by non-linear function $g(x)$.

$$
T L_{r}^{t}=g_{r}\left(Q_{r}^{t}, E L_{r+1}^{t}\right)
$$

\subsubsection{Reservoir high and low water level limits}

The default limits on the reservoir volumes are between the dry season control water and the normal operating level in non-flood season, while in flood season, the water level should be limited in a small fluctuation range around the flood control level.

$$
E L_{r, \min } \leq E L_{r}^{t} \leq E L_{r, \max }
$$

\subsubsection{Reservoir high and low release limits}

In order to ensure downstream navigation and the needs of ecological operation and to consider the maximum release capacity of the reservoir, there is a certain range within which the reservoir release should be.

$$
Q_{r, \min } \leq Q_{r}^{t} \leq Q_{r, \max }
$$

\subsubsection{System power demand constraints}

In general, the power demand is higher in the day time than at night so we denote the time varying demand as $D^{t}$. For each time interval, the total output of all the hydropower units should be equal or greater than the load demand. i.e.:

$$
\sum_{i=1}^{10} n_{i}^{t} P_{i}^{t} \geq D^{t}
$$

\subsubsection{Additional constraints on amount of water used}

One constraint added to the model for Case 2 is to limit the total amount of water used as shown in Eq. (15) below:

$$
\sum_{i=1}^{5} \sum_{i=1}^{5} n_{i}^{t} q_{i}^{t} \leq Q_{\text {actual }}
$$

Other constraints and the process are the same as the model described from Eq. (9)-(14).

All the constraints are handled by either of the two ways: one is to set certain limit conditions when producing initial population and evolving new generation. The other 
way is to check whether they are satisfied after the calculation and the infeasible solutions are filtered out by penalty function.

\section{Case study}

The procedures described in the preceding section were applied to the Three Gorges Project (TGP) cascade power system consisting of two cascade reservoirs and 47 hydro power units of 10 types.

\subsection{Overview of the TGP cascade power system}

TGP has the largest hydropower capacity in the world totaling $18,200 \mathrm{MW}$. Together with the GZB hydropower station $38 \mathrm{~km}$ downstream with a capacity of $2,715 \mathrm{MW}$, the TGP supplies electric energy for Central China, East China and the Guangdong province. Meanwhile, flood control is another significant function of the TGP-GZB. With flood control storage of $22.15 \times 10^{9} \mathrm{~m}^{3}$, the control area of the TGP-GZB occupies upper reaches of the Yangtze River, and thus has a great effect on the Middle-Lower Yangtze Region.

The TGP has two power plants, one on the Left Bank (LB) (2 types of units and total of 14 units) and the other on the Right Bank (RB) (3 types of units and total of 12 units). Each unit capacity of the 26 generators in the TGP is $70 \mathrm{MW}$, but they are from different manufacturers, and have different curves as shown in Fig.1. The GZB is a smaller storage facility with Er Jiang (EJ) and Da Jiang (DJ) power plants, housing 14 and 7 hydropower units respectively. There are 3 kinds of units among the 21 generators in the GZB, which have their most efficient power output at $12.5 \mathrm{MW}, 14 \mathrm{MW}$, and $17 \mathrm{MW}$, respectively. Computation of the head for each of the power plants is discussed in Eq. (3).

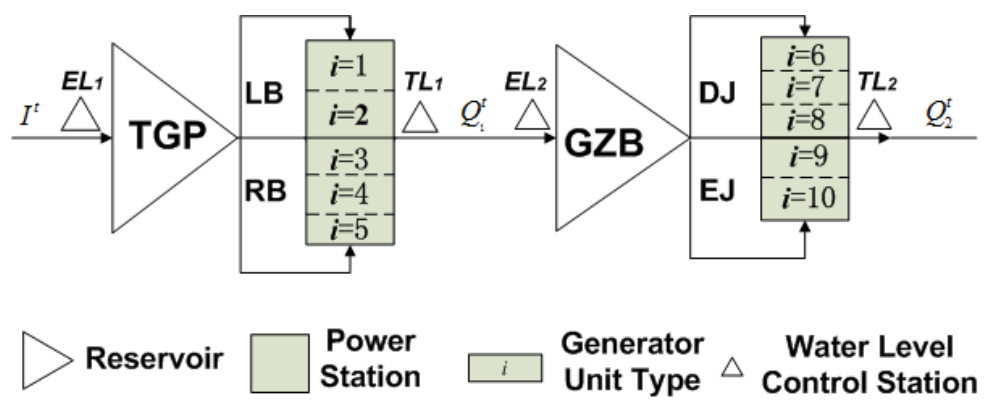

Fig.3. Schematic diagram of the TGP-GZB Cascade Hydropower Stations 
Fig.3 presents an installation sketch of various hydraulic hydropower units in each power plant, as well as the location sketch of the power plants and the water level control stations. Index $i$ is used to indicate different types of hydropower units, which is numbered later in Table 1.

Since the output characteristics vary among different types of hydropower units, and different plants provide different hydraulic conditions, the operation of the TGP-GZB system is complex. Both the complexity and enormous power generation capacity of the TGP-GZB imply there is considerable room for improvement over current operational policy.

Table 1 Number of different types of hydropower units as shown in Fig.3

\begin{tabular}{|c|c|c|c|c|c|c|c|c|c|c|}
\hline \multirow{2}{*}{$\begin{array}{c}\text { Stations } \\
i\end{array}$} & \multicolumn{3}{|c|}{ LB } & \multicolumn{2}{|c|}{$\mathrm{RB}$} & \multicolumn{2}{|c|}{ DJ } & \multicolumn{3}{|c|}{ EJ } \\
\hline & 1 & 2 & 3 & 4 & 5 & 6 & 7 & 8 & 9 & 10 \\
\hline Number & 8 & 6 & 4 & 4 & 4 & 13 & 1 & 4 & 2 & 1 \\
\hline
\end{tabular}

\subsection{Decision variables}

Fig.4 shows a typical daily load demand curve of the weekday in January of the year 2009. The figure shows that there are two peak load periods in a day, i.e. 9:00-11:00 and 17:00-21:00. During the night, the load curve is smooth and steady at a lower level. The load is a little lower than the peak load in noon time from 11:00 to 17:00. Thus, the schedule horizon can be divided into 5 intervals in non-flood season. 


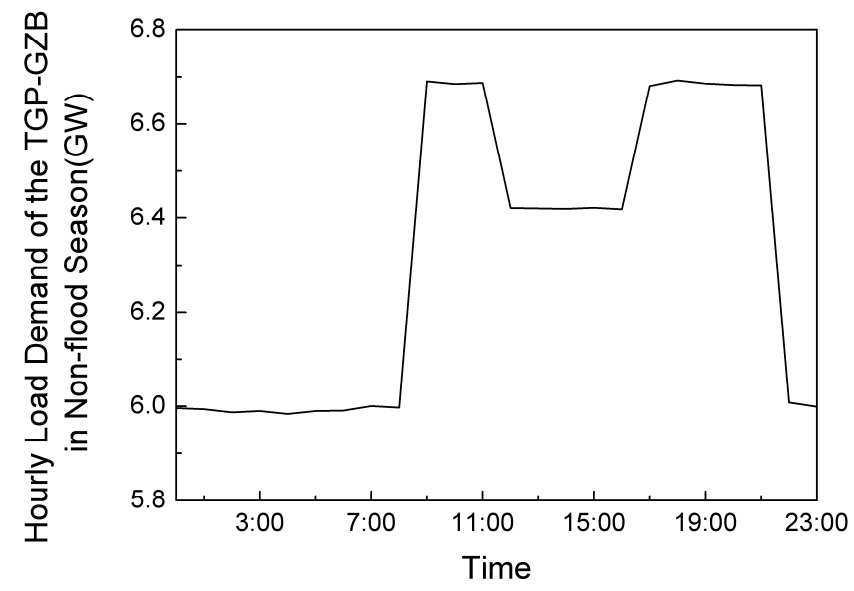

Fig.4. Typical daily load demand curve for the TGP-GZB cascade hydropower stations in non-flood season

The TGP has a large storage capacity. Thus small change of the water level results in great difference of the discharge. Hence, the outflow from the reservoir TGP and the GZB reservoir in each time period are chosen as the decision variables, which are expressed as follows:

$$
Q=\text { vector of all } Q_{r}^{t}
$$

which is a 10-D vector for this two reservoir system with 5 time periods.

\subsection{Implementation}

The input data for the model includes the load pattern of the system, the inflow to the TGP upstream. The parameters of the system of reservoirs model consist of the linear coefficients in the linear programming problem described by Eq. (2), the limits of water level in Eq. (12), the limits of release in Eq. (13), and the parameters used to describe the curves in Eq. (10) and Eq. (11).

First, a certain number of candidate solutions of the water distribution between the TGP and the GZB represented by Eq. (16) are generated randomly within the feasible region in Eq. (12) and Eq. (13) to be the initial population in the genetic algorithm. These candidate solutions consist of the release sequence of the TGP and the water level sequence of the reservoir GZB. (These same candidate solutions are used for initial points for all the heuristic search methods.) With the equality constraints from Eq. (9) to Eq. (11), the net water head can be calculated by Eq. (3). Thus, in the inner loop, the best 
allocation of the turbine discharge among different types of units is then determined. The output of the inner loop serves as the constraints in Eq. (14) for the outer loop so that the feasibility of the solution can be checked. Those feasible solutions are then compared with the objective function value in Eq. (7) or Eq. (8) as the standard for Case 1 or Case 2 respectively. The feasible solution with the best objective function value is then selected. The iteration of the heuristic optimization in outer loop continues to improve the objective function value in Eq. (7) or Eq. (8). Finally the optimum can be obtained when meeting the termination criteria.

\subsection{Parameter calibration}

\section{Inner loop:}

In this example, for the small reservoir GZB $(r=2)$, the discrete values of water head are $H_{r}^{t} \hat{\mathrm{I}}\{15.0,15.5, \ldots, 27.0\}$, and $i=6,7, \ldots, 10$. For the large reservoir TGP $(r=1)$, when reaching the normal water level $(175.0 \mathrm{~m})$, a $0.1 \mathrm{~m}$ difference in the water level means a difference of $1.0 \times 10^{8} \mathrm{~m}^{3}$ in the water volume. In order to simulate more accurately, the interval of the water head $H_{r}^{t}$ in Eq. (2) should be as small as possible, in this example, $72 \leq H_{r}^{t} \leq 113$ for the TGP $(r=1)$, so taking $0.1 \mathrm{~m}$ as discrete interval yields 410 linear coefficients for $C_{1 i}\left(H_{r}^{t}\right)$ and $C_{2 i}\left(H_{r}^{t}\right)$.

Li et al. (2013) has proven such linearization method has high accuracy to represent the power output of the TGP-GZB cascade system. In their research, the optimization is for long-term with higher variable dimensions, so the bivariate Taylor series expansion was applied to obtain a linear relationship between $Q$ and $H$ for the TGP to save computational cost. In this study, the water head are fixed by discretization for both the TGP and the GZB to avoid the uncertainty caused by Taylor series expansion.

\section{Outer loop:}

Seven different heuristic search algorithms are tested for the outer loop optimization, including SGA, IGA, SA, DDS, DYCORS, and SRBF. The total number of (objective) function evaluations is given for each algorithm, since this is the computationally expensive part of the analysis. The values of the algorithm parameters are discussed below.

The basic parameters of the SGA and IGA are the same: the individuals are mutated 
uniformly with the probability $P_{m}=0.1$, and the population at each iteration includes 100 individuals. The probability of crossover is $P_{c}=0.9$. The iteration terminated after 100 times.

For SA in this study, the initial probability of accepting uphill move is $P_{1}=0.95$. Hence, to approximate this probability, the initial temperature was set to $T_{0}=(-\operatorname{avg} \Delta \cos t) / \ln P_{1}=-6.50$, and the average cost (avg $\left.\Delta \cos t\right)$ was estimated by doing objective function evaluations at 500 values of the decision vector $u$. The temperature at the $g$-th iteration is $T_{g}=\alpha T_{g-1}$. The value of $\alpha$ is selected as $\alpha=0.999$ so that the probability of accepting an uphill move after 10,000 iterations is 0.01 . The constraints are first checked. An infeasible solution is rejected and a new random solution is generated until a feasible solution is found.

A default value of the only parameter $r$ in DDS is recommended as 0.2 , because this yields a sampling range that practically spans the normalized decision variable range. The normalization converts the original variable (which ranges from a minimum to a maximum that is specific for each variable) into a variable with a $[0,1]$ range so that all variables have an equal range and all variables are given equal weight in search. This is done internally in the DDS algorithm. Thus the DDS algorithm does not require extra simulations to tune algorithm parameters.

The population size of the PSO is set as same as GA, so there are 100 particles flying in each iteration, i.e., $S=100$ (swarm size); total number of iterations 100 . The personal and global best acceleration constants are both constant as $c_{l}=c_{2}=1.5$. Inertia weight is introduced to control the momentum of the particle by considering only a fraction of the previous motion direction in the calculation of the new velocity In this study, the inertia weight factor linearly decreases from an initial value $w_{0}=0.9$ to a final value $w_{n}=0.4$ as Ben Ghalia suggested (Ben Ghalia, 2008). The maximum velocity $V_{\max , j}$, which is used to arbitrarily limit the velocities of the particles, is set equal to the upper bound of the search range in dimension $j$.

The number of candidate points in each iteration for both the DYCORS and the SRBF are also set to be 100 , and the total iteration number is 100 . The radial basis function (RBF) interpolation model given below is used for both the DYCORS and the SRBF in this study. Given $n$ distinct points $x_{1}, \ldots, x_{n} \in \square^{d}$ where the function values $f\left(x_{1}\right), \ldots, f\left(x_{n}\right)$ are known, the RBF use an interpolant of the form 


$$
s_{n}(x)=\sum_{i=1}^{n} \lambda_{i} \phi\left(\left\|x-x_{i}\right\|\right)+p(x), \quad x \in \square^{d}
$$

where $\|\cdot\|$ is the Euclidean norm, $\lambda_{i} \in \square$ for $i=1, \ldots, n, \quad p(x)$ is a linear polynomial in $d$ variables, and $\phi$ has the cubic form: $\phi(r)=r^{3}$. Define the matrix $\Phi \in \square^{n \times n}$ by: $\Phi_{i j}:=\phi\left(\left\|x_{i}-x_{j}\right\|\right), i, j=1, \ldots, n$. Also, define the matrix $P \in \square^{n \times(d+1)}$ so that its $i$-th row is $\left[1, x_{i}^{\mathrm{T}}\right]$. Now, the cubic RBF model that interpolates the points $\left(x_{1}, f\left(x_{1}\right)\right), \ldots,\left(x_{n}, f\left(x_{n}\right)\right)$ is obtained by solving the system

$$
\left(\begin{array}{cc}
\Phi & P \\
P^{\mathrm{T}} & 0
\end{array}\right)\left(\begin{array}{l}
\lambda \\
c
\end{array}\right)=\left(\begin{array}{l}
F \\
0_{d+1}
\end{array}\right)
$$

where $F=\left(f\left(x_{1}\right), \ldots, f\left(x_{n}\right)\right)^{\mathrm{T}}, \lambda=\left(\lambda_{1}, \ldots, \lambda_{n}\right)^{\mathrm{T}} \in \square^{n}$ and $c=\left(c_{1}, \ldots, c_{d+1}\right)^{\mathrm{T}} \in \square^{d+1}$ consists of the coefficients for the linear polynomial $p(x)$.

\section{Results}

\subsection{Comparison of the efficiency of the algorithms}

Seven heuristic algorithms are compared in Fig.5 and Fig.6, the descriptions of which are given above. Recall that the heuristic optimization algorithm is used to solve the outer loop and in all cases the inner loop is solved by linear programming. Fig.5 compares the average objective function value for minimizing the amount of water used while satisfying the load demand for Case 1, using January $1^{\text {st }} 2009$ conditions for the example. The objective function of Case 1 is in Eq. (7), and constraints and computation process are described in Eq. (9) to Eq. (14). The algorithms comparisons of Case 2 are shown in Fig. 6 to find out how much extra power can be generated by the water saved. The objective of Case 2 is to maximize daily power generation with the actual amount of water used, as presented in Eq. (8).

Two different ways to generate initial solutions are also compared for both cases. One is making use of the actual operation, shown in Fig. 5(a) and Fig. 6(a). To be specific, for those single point-based methods, such as SA and DDS, the optimization starts with the actual operation; while for those population-based evolutionary algorithms, including SGA, IGA, PSO, DYCORS, and SRBF in this study, the actual operation is put into the initial populations as one of the initial solutions. The other way is to randomly generate the initial solutions without any guidance, shown in Fig. 5(b) and Fig. 6(b). It should be noted that with random seeds for Case 1, DDS is incapable of finding any 
feasible solution in all 20 trials.

The values plotted are the ratio between the average best values obtained from 20 trials for the specified algorithm after the specified number of objective function evaluations and the actual data. The starting points of all the trials are the same in Fig. 5(a) and Fig. 6(a), which is the actual operational data. Standard error of the mean (SEM) are also presented in Fig. (5) and Fig. (6) as the error bars, which graphically represent the variability of the specified algorithm in different trials.

For Case 1, the best optimization result among all the algorithms only use $94.4 \%$ of the water used in actual operation to generate the same power energy, which means $2.59 \times 10^{7} \mathrm{~m}^{3}$ water can be saved in the selected day; while for Case 2, the value of 1.045 in Fig.6 represents an increase in power production of 6.79 GWh more energy for the optimal policy over the historical policy using the same amount of water.

It needs to be clarified that the actual operation of the TGP-GZB system has been optimized by a real-time optimization system, so it has relatively small space to improve. Nevertheless, as one of the biggest hydropower systems in the world, the TGP and GZB projects have the capacity of $18,200 \mathrm{MW}$, and $2,715 \mathrm{MW}$, respectively, and an improvement of $5 \%$ means a capacity of around 105MW. Even though the improvements gained by optimizing both water usage and power generation appear to be marginal, a relatively large amount of water is saved and the additional the power generation with the same water used is also considerable. 


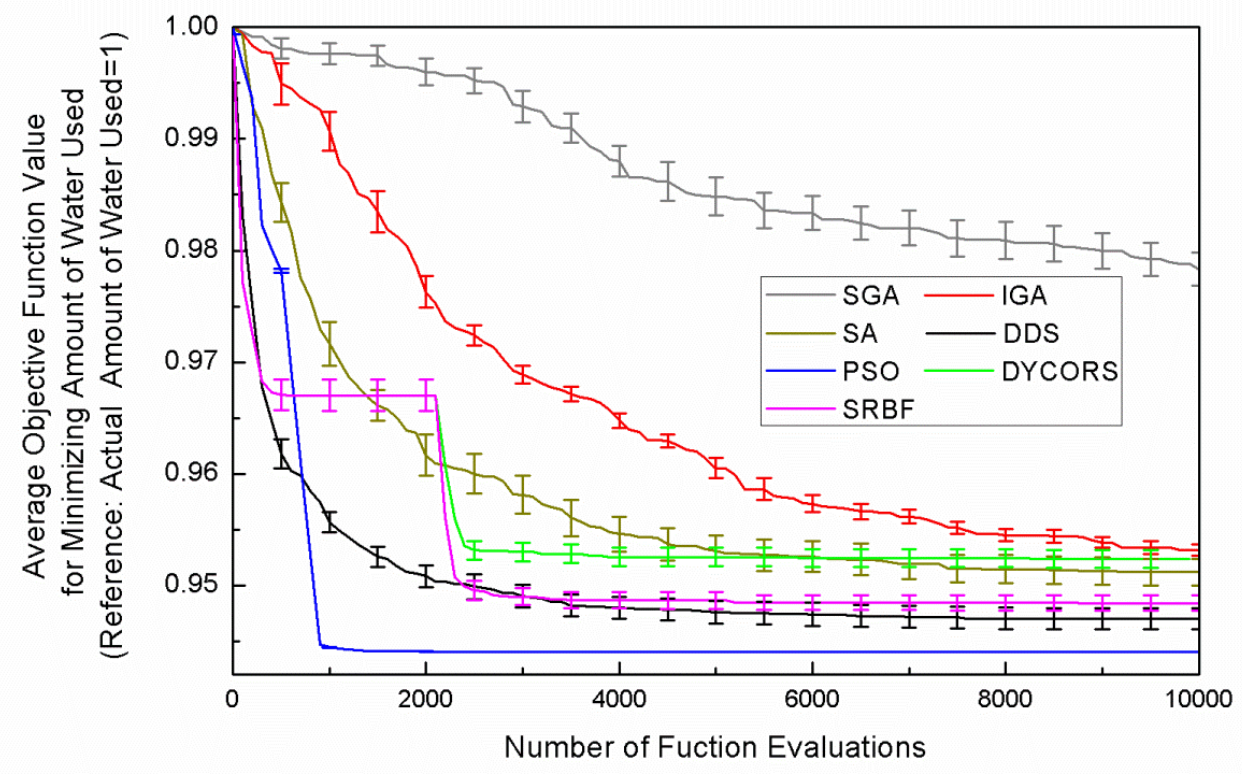

(a) With actual operations in the initial population

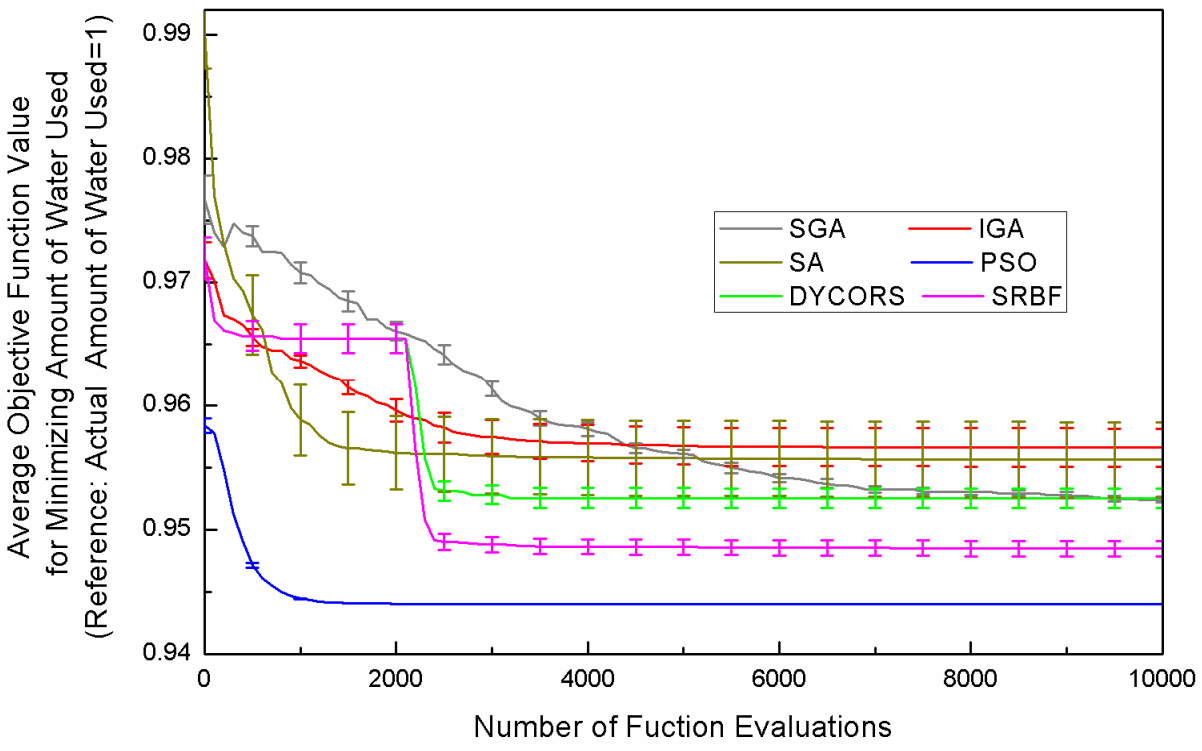

(b) With initial solutions generated randomly

Fig.5. Comparisons of different algorithms for the optimization problem in Case 1 on January $1^{\text {st }}$ 2009 in Eq. (7). Lowest curve is best for minimization. 


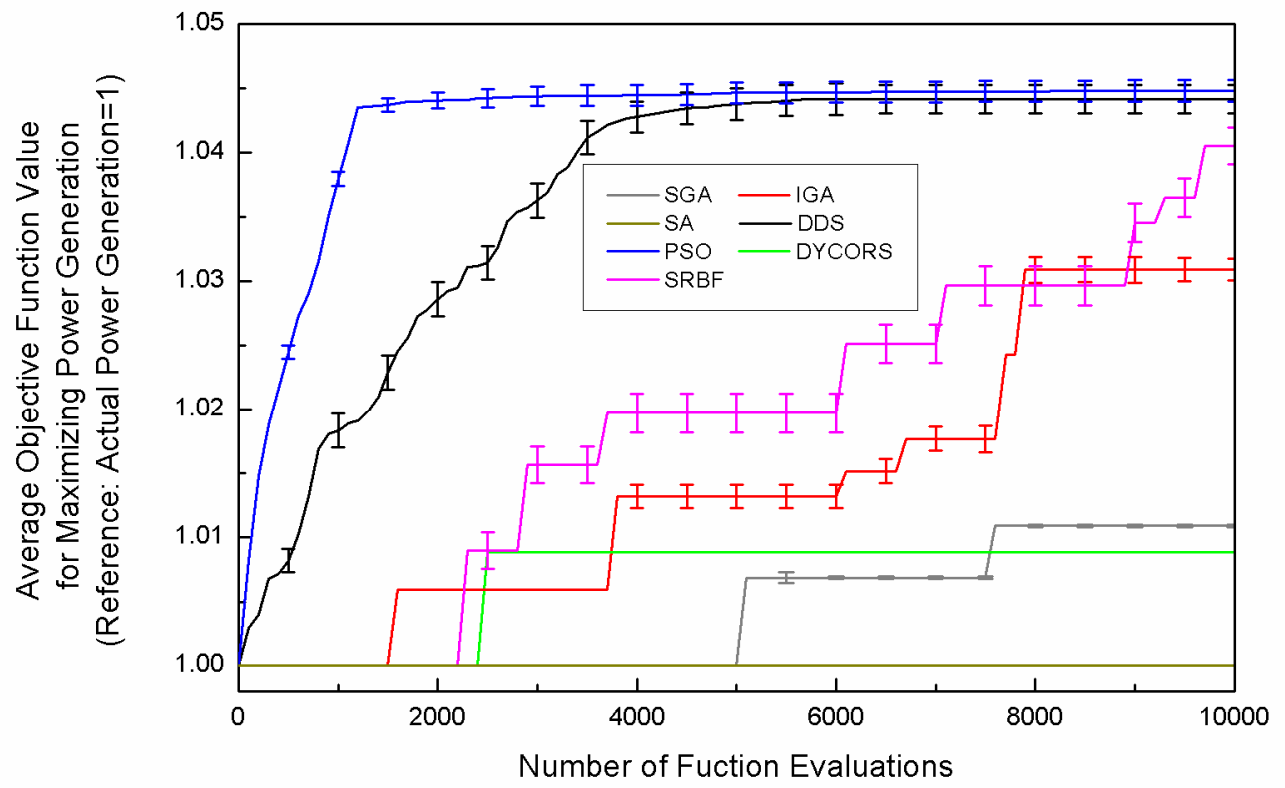

(a) With actual operations in the initial population

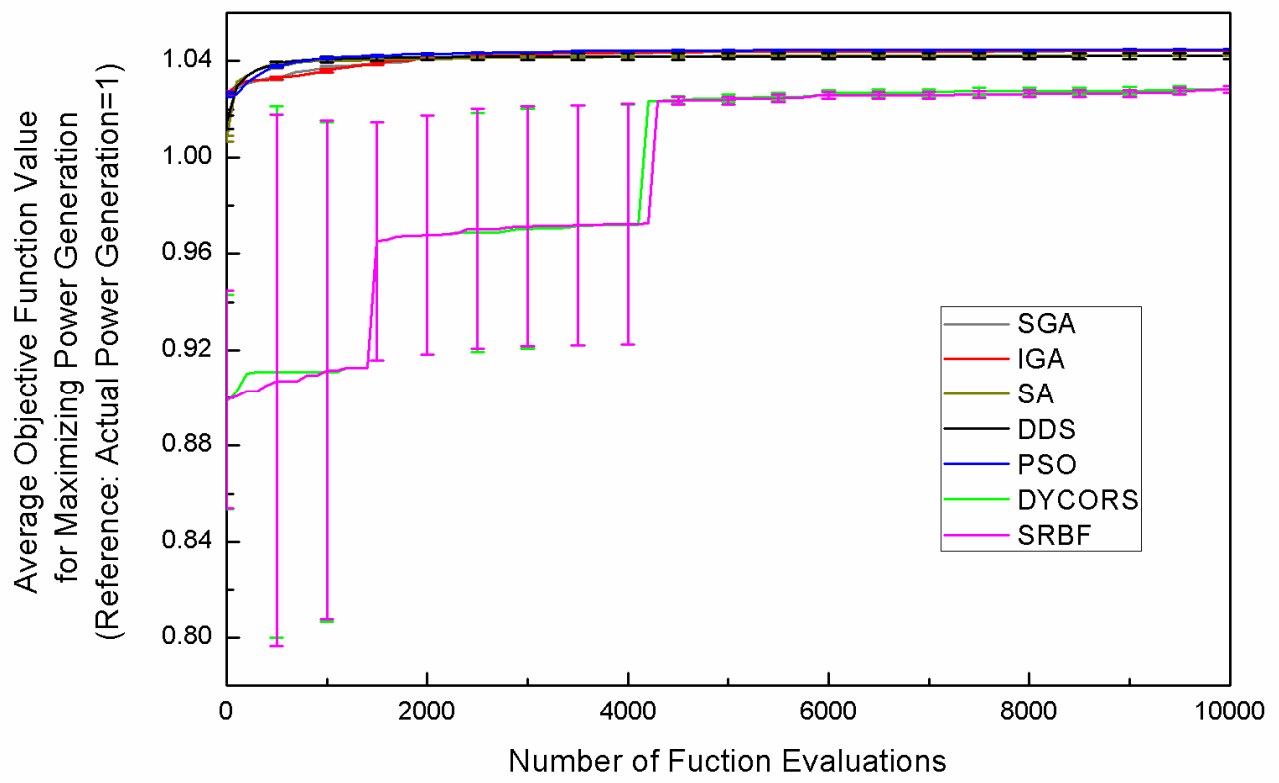

(b) With initial solutions generated randomly

Fig.6. Comparisons of different algorithms for the optimization problem in Case 2 on January $1^{\text {st }} 2009$ in Eq. (8) Curve is average over 20 trials per algorithm. Highest curve is best for maximization. 


\subsection{Comparison of the additional power generation of different types of generator units}

As mentioned above, there are 10 different types of power generator units in the TGP-GZB cascade hydropower stations, which have different characteristic curves describing the relationships among water head, turbine discharge and power output. The optimization result of maximizing the power generation by different algorithms is given in Fig.6.

PSO guided by the actual operation performs the best and produces totally about 1.045 times of the actual power generation, which means $6.79 \mathrm{GWh}$ extra power generation for one day compared to the historical output on January $1^{\text {st }}, 2009$ using the same input conditions.

Fig.7 shows the comparison of the amount of power output between the actual historical data A and the optimized results O by PSO for various types of units in each period.

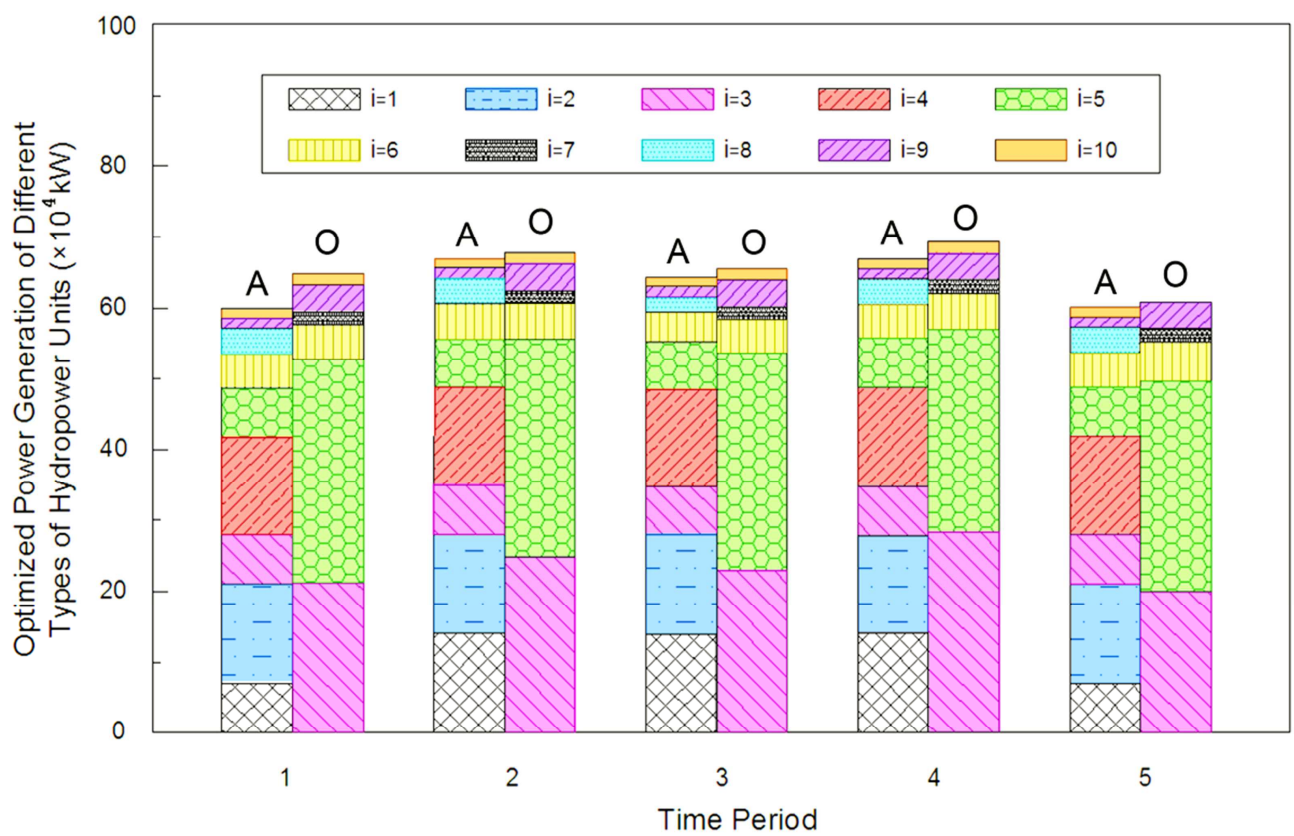

Fig.7. Comparison of total historical and optimized power output by different types of units in each time period on January $1^{\text {st }} 2009$ for Case 2. Optimized power is ' $O$ ' and actual historical power is 'A'. Both situations use the same amount of water. O produces $6.79 \mathrm{GWh}$ more energy in one day.

\subsection{Comparison of the Optimization in Each Month}


The flood season of the Yangzi River lasts from the mid-June to the end of September. The inflow to the TGP reservoir in flood season generally ranges from $15,000 \mathrm{~m}^{3} / \mathrm{s}$ to more than $50,000 \mathrm{~m}^{3} / \mathrm{s}$, and the inflow in non-flood season is less than $15,000 \mathrm{~m}^{3} / \mathrm{s}$. Besides, more power generation is needed in summer than winter; the beginning on peak demand in a day is earlier in summer than winter, and the duration time of peak demand is longer in summer than winter.

Although the load demand and the inflow differ from month to month, the load demand curve can be divided into 5 intervals in all seasons. Thus, the decision variables in the outer loop are still 10 dimensional for this two cascade reservoir system.

Table 2 presents the optimization results of the daily water used and daily power generation of a random day in each month. In flood season, the TGP reservoir has to maintain at a flood limit water level to prepare for the possible flood in the future. There is surplus water in operation in flood season. Hence, it is not necessary to minimize amount of water used in flood season, only the maximum power generation is taken into account from June to September.

Table 2(a). The optimization results of Case 1 for a random day in each month*

\begin{tabular}{|c|c|c|c|c|c|c|c|c|c|}
\hline \multicolumn{2}{|c|}{ Month } & 1 & 2 & 3 & 4 & 5 & 10 & 11 & 12 \\
\hline \multicolumn{2}{|c|}{ Actual water } & 463 & 467 & 588 & 603 & 655 & 638 & 532 & 480 \\
\hline \multirow{7}{*}{$\begin{array}{c}\text { Minimum } \\
\text { amount of water } \\
\text { used to meet the } \\
\text { load demand } \\
\text { (Case } 1) \\
\left(\times 10^{6} \mathrm{~m}^{3}\right)\end{array}$} & SA & 457 & 465 & 548 & 603 & 650 & 638 & 528 & 470 \\
\hline & SGA & 448 & 449 & 536 & 603 & 652 & 638 & 519 & 463 \\
\hline & IGA & 447 & 453 & 549 & 572 & 635 & 617 & 523 & 459 \\
\hline & DDS & 444 & 448 & 537 & 571 & 628 & 615 & 517 & 459 \\
\hline & DYCORS & 457 & 461 & 550 & 587 & 634 & 614 & 524 & 462 \\
\hline & SRBF & 444 & 447 & 537 & 579 & 630 & 622 & 521 & 458 \\
\hline & PSO & 437 & 441 & 532 & 565 & 628 & 608 & 511 & 450 \\
\hline \multicolumn{2}{|c|}{$\begin{array}{c}\text { Percentage of improvement of } \\
\text { the best algorithm }\end{array}$} & $5.6 \%$ & $5.6 \%$ & $9.5 \%$ & $6.3 \%$ & $4.1 \%$ & $4.7 \%$ & $3.9 \%$ & $6.3 \%$ \\
\hline
\end{tabular}

* The best algorithm in each month is in bold.

Table 2(b). The optimization results of Case 2 for the random day in each month* 


\begin{tabular}{|c|c|c|c|c|c|c|c|c|c|c|c|c|c|}
\hline \multicolumn{2}{|c|}{ Month } & 1 & 2 & 3 & 4 & 5 & 6 & 7 & 8 & 9 & 10 & 11 & 12 \\
\hline \multicolumn{2}{|c|}{$\begin{array}{l}\text { Actual power generation } \\
\qquad\left(\times 10^{8} \mathrm{kWh}\right)\end{array}$} & 1.51 & 1.51 & 1.81 & 1.82 & 3.90 & 3.38 & 4.61 & 4.38 & 3.28 & 2.00 & 1.74 & 1.56 \\
\hline \multirow{7}{*}{$\begin{array}{l}\text { Maximum } \\
\text { power } \\
\text { generation } \\
\text { with actual } \\
\text { water used } \\
(\text { Case } 2) \\
\left(\times 10^{8} \mathrm{kWh}\right)\end{array}$} & SA & 1.51 & 1.52 & 1.81 & 1.84 & 3.90 & 3.38 & 4.61 & 4.38 & 3.32 & 2.00 & 1.76 & 1.56 \\
\hline & SGA & 1.52 & 1.55 & 1.87 & 1.90 & 3.98 & 3.45 & 4.67 & 4.52 & 3.44 & 2.00 & 1.76 & 1.56 \\
\hline & IGA & 1.54 & 1.57 & 1.88 & 1.93 & 4.09 & 3.58 & 4.88 & 4.80 & 3.68 & 2.01 & 1.89 & 1.57 \\
\hline & DDS & 1.56 & 1.59 & 1.98 & 2.01 & 4.40 & 3.92 & 5.07 & 5.06 & 3.75 & 2.12 & 1.80 & 1.63 \\
\hline & DYCORS & 1.54 & 1.56 & 1.93 & 2.00 & 4.43 & 3.92 & 4.98 & 4.65 & 3.68 & 2.12 & 1.84 & 1.65 \\
\hline & SRBF & 1.54 & 1.57 & 1.95 & 2.02 & 4.45 & 3.90 & 5.04 & 4.97 & 3.87 & 2.10 & 1.90 & 1.61 \\
\hline & PSO & 1.58 & 1.58 & 1.98 & 2.03 & 4.47 & 3.95 & 5.06 & 5.06 & 3.89 & 2.15 & 1.90 & 1.67 \\
\hline \multicolumn{2}{|c|}{$\begin{array}{l}\text { Percentage of } \\
\text { improvement of the best } \\
\text { algorithm }\end{array}$} & $4.5 \%$ & $5.3 \%$ & $9.4 \%$ & $\begin{array}{c}11.5 \\
\%\end{array}$ & $\begin{array}{c}14.6 \\
\%\end{array}$ & $\begin{array}{c}16.9 \\
\%\end{array}$ & $\begin{array}{c}9.98 \\
\%\end{array}$ & $\begin{array}{c}15.5 \\
\%\end{array}$ & $\begin{array}{c}18.6 \\
\%\end{array}$ & $7.5 \%$ & $9.2 \%$ & $\begin{array}{c}7.1 \\
\%\end{array}$ \\
\hline
\end{tabular}

* The best algorithm in each month is in bold.

\section{Discussion}

As pointed out by Maier et al. (2014), despite advances in computing power and parallel computing, the need for reducing the size of the search space is still as acute as it was 30 years ago, as we attempt to solve larger and larger problems. In this work, we intended to reduce the search space size of heuristic algorithms for a real-world multi-reservoir optimization problem by decomposing it into two coupled sub-problems: (i) outer loop: optimizing water dispatching scenario among reservoirs; (ii) inner loop: for each reservoir, optimizing hydro scheduling plan for different hydropower units. The hierarchical or decomposing concept embodied in this study is much more important for search space reduction than the model itself. The key operation, which is also a main source of difficulty for such methods, is to decouple the original problem with as small loss of information as possible. Another considerable issue when developing such models is that generic models are much more desirable than case-study specific approaches. The hierarchical model presented in this paper is based on the underlying physical mechanisms, which makes it both easy-to-understand and universal.

The TGP-GZB hydropower plants are complex and representative of multi-reservoir systems with heterogeneous units. The application to this real-world problem proves the effectiveness of the proposed method for dimensionality reduction. Seven different heuristic algorithms are tested in the case study, and the results are discussed as below:

(1) Characteristics of different heuristic algorithms

SGA almost performs the worst when given guidance. However, with initial 
population generated randomly, SGA keeps improving, and the final best value is on average level. The premature convergence thus seems prominent for SGA, i.e., once a certain high-quality solution appears, the evolution will quickly converged to it, and has poor ability to jump out of it. Hence, the initial population for SGA should be as diverse as possible.

The performance of the IGA is stable but not outstanding. For all the cases we considered, IGA is capable to obtain a relatively good optimum through continuous optimization. The main difference between IGA and SGA lies in the mechanism of crossover and mutation. IGA restricts its search only to the permissible values of the variables, which helps reducing the search effort in converging to the optimum solution. While for the random seeds, such a restriction is counterproductive in our study. Since the variation range of each gene of the individual is decreased compared to SGA, the optimization converges rapidly to a certain optimum.

As a single point-based algorithm, the variation range of solutions in SA is usually not as large as other population-based algorithms, but an optimum on average level can still be found by SA. It is noticeable that, in Case 2 there is an additional constraint in Eq. (15) over the constraints for Case 1, and this constraint leads to a much larger fraction of infeasible points generated in the search. This difference seems especially to affect SA, which cannot improve at all when given the actual operation as the starting point. However, when the initial solution is generated randomly, only 1 trial over the total 20 trials cannot find feasible solutions. This also indicates that the performance of SA relies on the initial solution to a great extent.

Although DDS is a recently developed heuristic algorithm with simple idea and implementation, its performance is still satisfying as a single point-based algorithm. When given a high-quality starting point, DDS maintains continuous improvements. Nevertheless, DDS shows strong dependence on the starting point like SA. In some extreme cases, such as Case 1, DDS is unable to obtain any feasible solution in any trial when starting from random points.

PSO performs the best almost in all the cases in this study. There are two reasons for this (1) PSO has the advantage of population optimization, which not only increase the diversity of solutions, but also decrease the dependence on the initial solutions; (2) the evolution of the solutions in the case of the PSO algorithm relies less on the previous 
generation compared to other population-based algorithms, and this helps to have a more diverse set of solutions.

DYCORS and SRBF are both based on the response surface, which perform quite well for Case 1, but for Case 2, both of them perform poorly, especially when the initial population is generated randomly. DYCORS and SRBF have their own way to generate the candidate points according to the previous results to make the population as diverse as possible, thus the diversity of the algorithms is distinct. But it still has the problem of being stuck in a local optimum.

Generally, although some algorithms show great dependence on the quality of initial points and some do not, an initial population with high-quality helps to get better optimization results for almost all the algorithms. Hence, when using the proposed hierarchical model to solve multi-reservoir optimization problems, it is strongly recommended to use the actual operation or other operational experience as guidance; while for real-time optimization without any actual data, the reservoir operational chart can be used as the reference.

(2) Characteristics of different types of hydropower units

With Case 2, both $\mathrm{O}$ and $\mathrm{A}$ have the same total water release discharge (equaling the actual historical discharge of January $\left.1^{\text {st }}, 2009\right)$ but $\mathrm{O}$ produces more energy. Not all types of hydro generating units are put into operation in the optimal scheduling. Only those that are more efficient under the current water head are in operation. In this case, units 3 and 5 are preferred due to their higher efficiency under the water head given. In actual operation, there are some other factors that have to be considered, such as the start-up and shut-down costs as well as the unit maintenance. The generating units cannot always run with the maximum possible output, which affects the service life of units. The optimized results provide a theoretical maximum power output with the given flow condition.

With more optimization data, a knowledge base of the most efficient type of units under a certain hydraulic condition can be established, and provide the reference for actual operation.

(3) Optimization in different seasons

For different months, the percentage of the difference between the optimized power generation and the actual power generation ranges from about 3\% to almost $15 \%$. There 
is a larger improved room in flood season than in non-flood season. The reason for such a remarkable improvement is that the optimal solution found by linear programming is always at the vertexes of the feasible region, which mean the hydro units generate the possible maximum power output with current water head and turbine discharge limitation. In flood season, unlimited water discharge lead to the maximum power output under the water head. Thus, although the water head has to be lowered for flood in flood season, the power generation can be higher.

\section{Conclusion}

In order to reduce the search space size of real-world problems, this paper proposed a hierarchical model for the optimization of multi-reservoir systems with heterogeneous hydropower units. The optimization problem is decoupled into two loops: (i) outer loop: multi-reservoir optimization; and (ii) inner loop: optimal unit scheduling. Heuristic algorithms and linear programming are used for the two loops respectively. Alternative objectives of minimizing water used and maximizing power generation are considered. Taking the Three Gorges system as a study case, seven heuristic algorithms are tested for the outer loop. The results prove the model proposed is effective to reduce the search space size of such a complex system, and also provides guidance for algorithm selection, as well as reservoir operation.

In summary, the high dimensionality of the decision variables in real-world problems hinders the application of heuristic algorithms. Decomposing the original problems into coupled sub-problems is an effective way to reduce dimensionality. Although there have been previous studies on hierarchical models for reservoir optimization, our approach is unique for considering the unit scheduling problem in multi-reservoir systems. The mechanism of the decomposition has been demonstrated based on the reservoir operational background, and the effectiveness of the method is also proved by real-world application. The use of the proposed model for other real world hydropower systems is highly encouraged.

\section{Acknowledgements}

This research was supported by National Natural Science Foundation of China((Grant No.51409248), and the Open Research Fund of State Key Laboratory of 
Simulation and Regulation of Water Cycle in River Basin (China Institute of Water Resources and Hydropower Research)(Grant No.WHR-SKL-201409). Prof. Shoemaker's time was supported in part by NSF grants CBET 075675, EAR 0711491, and CCF

1116298. The authors would like to thank both the editor and the four anonymous reviewers for their valuable comments.

\section{References}

Arce, A., Ohishi T., Soares S. (2002).Optimal dispatch of generating units of the Itaipú hydroelectric plant. IEEE Transactions on Power Systems. 17,154-158.

Barros, M. T., Tsai F. T., Yang S.-1., Lopes J. E., Yeh W. W. (2003).Optimization of large-scale hydropower system operations. Journal of Water Resources Planning \& Management. 129,178-188.

Bavafa, M., Monsef H., Navidi N. (2009).A new hybrid approach for unit commitment using lagrangian relaxation combined with evolutionary and quadratic programming. Asia-Pacific Power and Energy Engineering Conference,IEEE, pp. 2134-2139.

Ben Ghalia, M. (2008).Particle swarm optimization with an improved exploration-exploitation balance, $51^{\text {st }}$ Midwest Symposium on Circuits and Systems, IEEE, pp. 759-762.

Borghetti, A., D'Ambrosio C., Lodi A., Martello S. (2008).An MILP approach for short-term hydro scheduling and unit commitment with head-dependent reservoir. IEEE Transactions on Power Systems. 23,1115-1124. Cai, X., McKinney D. C., Lasdon L. S. (2001).Solving nonlinear water management models using a combined genetic algorithm and linear programming approach. Advances in Water Resources. 24,667-676.

Castelletti, A., Lotov A. V., Soncini-Sessa R. (2010).Visualization-based multi-objective improvement of environmental decision-making using linearization of response surfaces. Environmental Modelling \& Software. 25,1552-1564.

Chang, L. C., Chang F. J., Wang K. W., Dai S. Y. (2010).Constrained genetic algorithms for optimizing multi-use reservoir operation. Journal of Hydrology. 390,66-74.

Deb, K. (2000).An efficient constraint handling method for genetic algorithms. Computer Methods in Applied Mechanics \& Engineering. 186,311-338.

Deb, K., Agrawal R. B. (1995).Simulated binary crossover for continuous search space. Complex Systems. 9,115-148.

Deb, K., Goyal M. (1996).A combined genetic adaptive search (GeneAS) for engineering design. Computer Science and Informatics. 26,30-45.

Diniz, A. L., Esteves P. P. I., Sagastizabal C. A. (2007).A mathematical model for the efficiency curves of hydroelectric units, Power Engineering Society General Meeting, IEEE, pp. 1-7.

Duo, H. Z., Sasaki H., Nagata T., Fujita H. (1999).A solution for unit commitment using Lagrangian relaxation combined with evolutionary programming. Electric Power Systems Research. 51,71-77.

El-Hawary, M. E., Christensen G. S. (1979).Optimal economic operation of electric power systems. Academic Press, New York.

Finardi, E. C., Scuzziato M. R. (2014).A comparative analysis of different dual problems in the Lagrangian Relaxation context for solving the Hydro Unit Commitment problem. Electric Power Systems Research. 107,221-229.

Gil, E., Bustos J., Rudnick H. (2003).Short-term hydrothermal generation scheduling model using a genetic algorithm. IEEE Transactions on Power Systems. 18,1256-1264.

Grygier, J. C., Stedinger J. R. (1985).Algorithms for optimizing hydropower system operation. Water Resources Research. 21,1-10.

Gutmann, H. M. (2001).A radial basis function method for global optimization. Journal of Global Optimization. 19,201-227.

Kennedy, J., Eberhart R. (1995).Particle swarm optimization, International Conference on Neural Networks Proceedings, IEEE. pp. 1942-1948. 
Kirkpatrick, S. (1984).Optimization by simulated annealing: Quantitative studies. Journal of Statistical Physics. 34,975-986.

Labadie, J. W. (2004).Optimal operation of multireservoir systems: State-of-the-art review. Journal of Water Resources Planning \& Management. 130,93-111.

Li, F. F., Wei J. H., Fu X. D., Wan X. Y. (2012).An effective approach to long-term optimal operation of large-scale reservoir systems: case study of the Three Gorges system. Water Resources Management. 26,4073-4090.

Maier, H. R., Kapelan Z., Kasprzyk J., Kollat J., Matott L. S., Cunha M. C., Dandy G. C., Gibbs M. S., Keedwell E., Marchi A., Ostfeld A., Savic D., Solomatine D. P., Vrugt J. A., Zecchin A. C., Minsker B. S., Barbour E. J., Kuczera G., Pasha F. (2014).Evolutionary algorithms and other metaheuristics in water resources: current status, research challenges and future directions. Environmental Modelling and Software. 62,271-299.

Malekmohammadi, B., Kerachian R., Zahraie B. (2009).Developing monthly operating rules for a cascade system of reservoirs: Application of Bayesian Networks. Environmental Modelling \& Software. 24,1420-1432.

Naresh, R., Sharma J. (2000).Hydro system scheduling using ANN approach. IEEE Transactions on Power Systems. 15,388-395.

Orero, S., Irving M. (1998).A genetic algorithm modelling framework and solution technique for short term optimal hydrothermal scheduling. IEEE Transactions on Power Systems. 13,501-518.

Piekutowski, M. R., Litwinowicz T., Frowd R. J. (1994).Optimal short-term scheduling for a large-scale cascaded hydro system. IEEE Transactions on Power Systems. 9,805-811.

Ponrajah, R. A., Witherspoon J., Galiana F. D. (1998).Systems to optimise conversion efficiencies at Ontario Hydro's hydroelectric plants. IEEE Transactions on Power Systems. 13,1044-1050.

Powell, M. J. D. (1992) The theory of radial basis function approximation in 1990, in: Light, W. (ed.), Advances in Numerical Analysis, Vol 2: Wavelets, Subdivision Algorithms and Radial Basis Functions, Oxford University Press, pp. 105-210.

Powell, M.J.D. (1999), Recent research at Cambridge on radial basis functions, in: M. Müller, M. Buhmann, D. Mache and M. Felten (eds), New Developments in Approximation Theory, International Series of Numerical Mathematics, Vol 132, Birkhauser Verlag, Basel, pp. 215-232.Regis, R. G., Shoemaker C. A. (2007).A stochastic radial basis function method for the global optimization of expensive functions. Informs Journal on Computing. 19,497-509.

Regis, R. G., Shoemaker C. A. (2013).Combining radial basis function surrogates and dynamic coordinate search in high-dimensional expensive black-box optimization. Engineering Optimization. 45,529-555.

Saad, M., Bigras P., Turgeon A., Duquette R. (1996).Fuzzy learning decomposition for the scheduling of hydroelectric power systems. Water Resources Research. 32,179-186. Singh, A. (2011).Global optimization of computationally expensive hydrologic simulation models, Cornell University.

Sulis, A. (2009).GRID computing approach for multireservoir operating rules with uncertainty. Environmental Modelling \& Software. 24,859-864.

Tolson, B. A., Shoemaker C. A. (2007).Dynamically dimensioned search algorithm for computationally efficient watershed model calibration. Water Resources Research. 43.

Tong, B., Zhai Q. Z., Guan X. H. (2013).An MILP based formulation for short-term hydro generation scheduling with analysis of the linearization effects on solution feasibility. IEEE Transactions on Power Systems. 28,3588-3599.

Tospornsampan, J., Kita I., Ishii M., Kitamura Y. (2005).Optimization of a multiple reservoir system using a simulated annealing-A case study in the Mae Klong system, Thailand. Paddy and Water Environment. 3,137-147.

Wakamori, F., Masui S., Morita K., Sugiyama T. (1982).Layered network model approach to optimal daily hydro scheduling. IEEE Transactions on Power Apparatus and Systems. 101,3310-3314. Wolpert, D. H., Macready W. G. (1997).No free lunch theorems for optimization. IEEE Transactions on Evolutionary Computation. 1,67-82.

Wong, K., Wong Y. (1994).Short-term hydrothermal scheduling part. I. Simulated annealing approach. IEE Proceedings-Generation, Transmission and Distribution. 141,497-501.

Yang, P. C., Yang H. T., Huang C. L. (1996).Scheduling short-term hydrothermal generation using evolutionary programming techniques, IEE Proceedings-Generation, Transmission and Distribution, IET. pp. 371-376. 
Yeh, W. W. G., Becker L., Hua S. Q., Wen D. P., Liu J. M. (1992).Optimization of real-time hydrothermal system operation. Journal of Water Resources Planning \& Management. 118,636-653.

Yi, J., Labadie J. W., Stitt S. (2003).Dynamic optimal unit commitment and loading in hydropower systems. Journal of Water Resources Planning \& Management. 129,388-398. 